\title{
Asparaginase activity monitoring and management of asparaginase hypersensitivity reactions in Canada
}

\author{
Meghan Pike ${ }^{1}$, Tamara MacDonald ${ }^{1}$, and Ketan Kulkarni ${ }^{1}$ \\ ${ }^{1}$ IWK Health Centre
}

February 24, 2021

\begin{abstract}
Background Pegaspargase (PEG), can cause anti-asparaginase (ASP) antibody formation, which can decrease its effectiveness without causing any clinically-apparent reaction (CAR) (silent inactivation [SI]). When a patient has SI, a switch to Erwinia ASP is warranted, but there is currently a global shortage of Erwinia. Up to 30\% of patients receiving PEG will experience a CAR, while only $8 \%$ of patients will experience SI; the only way to distinguish between these is by measuring an ASP level. However, routine ASP monitoring is not currently standard of care at all Canadian centres. This study aims to identify variations in practice regarding ASP monitoring and Erwinia use. Procedure A 21-item survey was developed using OPINIO software and distributed to all Pediatric Hematology-Oncologists in Canada from Feb-Oct 2020. Results Respondents represented 15 hospitals across each region of Canada (response rate=52\%). Only $39.2 \%$ of respondents reported routinely measuring ASP levels, yet $53 \%$ of respondents have modified therapy from PEG to Erwinia in up to half of their patients. The most common reason for not measuring ASP levels was not knowing how to use levels clinically (25.5\%). There was variation in the timing of levels and their target. Conclusions We identified substantial variation in ASP activity monitoring practices across Canada. Therefore, future research should aim to develop a national practice guideline on ASP activity monitoring.
\end{abstract}

Meghan Pike, MD

Subspecialty Resident, Pediatric Hematology-Oncology

IWK Health Centre/Dalhousie University

5850/5980 University Avenue

Halifax, Nova Scotia

B3K $6 \mathrm{R} 8$

phone: (902) 266-2790

fax: (902) 470-7216

meghan.pike@dal.ca

February $20^{\text {th }}, 2021$

Dear Dr. Newburger,

Thank you for considering our manuscript, entitled Asparaginase activity monitoring and management of asparaginase hypersensitivity reactions in Canada, for publication as a Research Article in Pediatric Blood and Cancer. Myself, Meghan Pike MD and my co-authors, Tamara MacDonald PharmD and Ketan Kulkarni MD FRCPC feel this study provides valuable information about the use of asparaginase and asparaginase activity level monitoring in Canada. 
This survey study, which had responses from physicians in every province in Canada, identifies that only $39.2 \%$ of pediatric oncologists in Canada routinely measure asparaginase levels. The most common reason for this not knowing how to use levels clinically. There is also variation in the timing of levels and their target(s). The survey also identified inconsistency of use of Erwinia and the authors feel that consistent asparaginase activity level monitoring may decrease the frequency of switching asparaginase formulations from pegaspargase toErwinia, which is especially important in light of the current global Erwinia shortage.The information provided by this study informs us of the need for a pan-Canadian guideline regarding asparaginase activity level monitoring and management of asparaginase reactions, which the authors intend to develop.

This manuscript has not been submitted elsewhere nor has it been previously published. The authors do not have any conflict of interest relating to this study.

All authors have contributed to the manuscript in significant ways, have reviewed and agreed upon the manuscript content. Three potential reviewers who are experts in this field without any identified conflict of interest include Dr. Sunil Desai, Dr. Jim Whitlock, Dr. Sarah Alexander.

Sincerely,

Meghan Pike, MD

Corresponding Author

Asparaginase activity monitoring and management of asparaginase hypersensitivity reactions in Canada

M Pike, T MacDonald, K Kulkarni

Meghan Pike, MD

Department of Pediatrics/Division of Pediatric Hematology-Oncology

Dalhousie University/IWK Health Centre

5850/5980 University Avenue

PO Box 9700

Halifax, Nova Scotia

B3K 6 R8

phone: (902) 266-2790

fax: (902) 470-7216

meghan.pike@dal.ca

Ketan Kulkarni, MD

Department of Pediatrics/Division of Pediatric Hematology-Oncology

Dalhousie University/IWK Health Centre

5850/5980 University Avenue

PO Box 9700

Halifax, Nova Scotia

B3K $6 \mathrm{R} 8$

(902) 470-3795 
ketan.kulkarni@iwk.nshealth.ca

Tamara MacDonald, PharmD (Corresponding Author)

Clinical Pharmacy Specialist Hematology/Oncology

Faculties of Medicine and Health Professions,

Dalhousie University/IWK Health Centre

5850/5980 University Avenue

PO Box 9700

Halifax, Nova Scotia

B3K $6 \mathrm{R} 8$

(902) 470-6474

tamara.macdonald@iwk.nshealth.ca

WORD COUNT: ABSTRACT: 219 words

WORD COUNT: MAIN TEXT: 2806 words

Number of Tables: 2

Number of Figures : 0

Number of Supporting Information Files: 1

Short Running Title: Asparaginase activity monitoring in Canada

Data Availability Statement: Data available on request from the authors.

Keywords: asparaginase, TDM, acute lymphoblastic leukemia, survey, hypersensitivity, Erwinia

\begin{tabular}{ll}
\hline Abbreviation & Full Term or Phrase \\
\hline ASP & Asparaginase \\
PEG & Pegaspargase \\
CAR & Clinically-apparent reaction \\
SI & Silent inactivation \\
CLIA & Clinical Laboratory Improvement Amendments \\
COG & Children's Oncology Group \\
CTCAE & Common Terminology Criteria for Adverse Events \\
SOP & Standards of Practice \\
NCCN & National Comprehensive Cancer Network \\
\hline
\end{tabular}

\section{Background}

Pegaspargase (PEG), can cause anti-asparaginase (ASP) antibody formation, which can decrease its effectiveness without causing any clinically-apparent reaction (CAR) (silent inactivation [SI]). When a patient has SI, a switch to Erwinia ASP is warranted, but there is currently a global shortage of Erwinia . Up to $30 \%$ of patients receiving PEG will experience a CAR, while only $8 \%$ of patients will experience SI; the only way to distinguish between these is by measuring an ASP level. However, routine ASP monitoring is not currently standard of care at all Canadian centres. This study aims to identify variations in practice regarding ASP monitoring and Erwinia use.

Procedure 
A 21-item survey was developed using OPINIO software and distributed to all Pediatric HematologyOncologists in Canada from Feb-Oct 2020.

Results

Respondents represented 15 hospitals across each region of Canada (response rate $=52 \%$ ). Only $39.2 \%$ of respondents reported routinely measuring ASP levels, yet $53 \%$ of respondents have modified therapy from PEG to Erwinia in up to half of their patients. The most common reason for not measuring ASP levels was not knowing how to use levels clinically $(25.5 \%)$. There was variation in the timing of levels and their target.

\section{Conclusions}

We identified substantial variation in ASP activity monitoring practices across Canada. Therefore, future research should aim to develop a national practice guideline on ASP activity monitoring.

\section{Introduction}

Asparaginase is integral in the treatment of pediatric leukemia/lymphoma. Asparaginase levels can be measured in serum as a surrogate marker for asparagine depletion and resultant leukemic cell death. Monitoring of serum asparaginase levels has been validated as a method of quantifying the efficacy of asparaginase formulations.

Trough asparaginase levels of $<0.1 \mathrm{IU} / \mathrm{mL}$ are likely subtherapeutic and may reflect the presence of antiasparaginase antibodies in the patient's blood. Pegaspargase (pegylated asparaginase) and Erwinia asparaginase formulations are available in Canada. Since pegaspargase is derived from E.coli, it is highly immunogenic and can cause clinically apparent hypersensitivity reactions (CARs) (i.e patient is reacting to the asparaginase portion of the drug) during or following administration [1]. Pegaspargase is asparaginase linked to polyethylene glycol which is compound that can itself cause an immune response. Immune-mediated reactions, both clinically apparent and subclinical, can contribute to the development of anti-asparaginase antibodies resulting in loss of therapeutic effect and reduced asparaginase serum levels. When this inactivation is subclinical it is termed silent inactivation (SI). SI has been reported in up to $8 \%$ of children who receive pegaspargase [2].

The presence of subtherapeutic levels or SI justifies a switch of asparaginase formulations from pegaspargase to Erwiniaasparaginase. Antibody cross-reactivity does not occur in patients treated with Erwinia who have neutralizing antibodies to pegaspargase, which allows for patients to achieve therapeutic levels of asparaginase with Erwinia . Erwinia is also less immunogenic than pegaspargase and thought to contribute less to anti-asparaginase antibody formation without compromising overall survival [2]. However, pegaspargase is preferred first-line asErwinia is less potent, more expensive and requires multiple injections, which impacts the practical administration of Erwiniaand the quality of life of patients.

Up to $30 \%$ of patients will develop clinical symptoms during pegaspargase infusion, such as flushing, tachycardia or hives [3]. These symptoms can be representative of an immune-mediated CAR (associated with a risk of low levels) or a non-immune mediated hypersensitivity/infusion-related reaction (not associated with low levels). These phenomena can be nearly impossible to distinguish clinically. The only way to reliably differentiate between an immune-mediated CAR and an infusion related reaction is by measuring asparaginase levels. Otherwise, patients with infusion related reactions to pegaspargase may be switched to Erwinia despite adequate asparaginase levels with pegasparagase.

There is concern that the use of clinical symptoms alone likely overestimates the true incidence of reactions to asparaginase and that of low serum levels. This is especially important to consider in light of the global shortage of Erwinia [3].

Despite this, routine asparaginase monitoring is not considered standard of care at all Canadian centers for reasons that are not entirely clear in the literature. Previously, there has been a lack of a widely available validated assay. However, Clinical Laboratory Improvement Amendments (CLIA)-certified laboratories offer asparaginase testing on serum/plasma specimens. Regardless, academic centers in Canada appear to be 
varied in their approach to measuring levels and there are different clinical practices regarding when to switch asparaginase preparations, when to order asparaginase levels and how to use those levels to guide management.

The objective of this study is to identify variations in individual practice and experience regarding asparaginase activity monitoring and the management of clinical asparaginase reactions across Canada using a survey. This study will describe: (1) management of reactions, (2) use of asparaginase activity monitoring , (3) reasoning for modifying, discontinuing or switching asparaginase preparations from pegaspargase to Erwinia asparaginase.

\section{Methods}

\section{Development of the survey}

This is a prospective observational study using a survey distributed to all Pediatric Hematology-Oncology physicians and subspecialty residents in Canada from February-October 2020. Authors developed a survey that consists of 19 multiple choice questions (see Supporting Information) 3 questions collect demographic information, 10 questions address the use and interpretation of asparaginase levels and 6 questions address the respondents' clinical experience with hypersensitivity reactions and modifying asparaginase formulations. A table defining the National Cancer Institute Common Terminology Criteria for Adverse Events (CTCAE) v.5.0 [4] was included in the survey for reference.

For 11 questions, respondents were asked to select one answer. For the remaining 8 questions, respondents were asked to select all answers that applied (see Supporting Information).

\section{Piloting survey at local institution}

We piloted the survey in a paper-based format to Pediatric Hematology-Oncology physicians $(\mathrm{n}=5)$ and subspecialty residents $(n=1)$ at the IWK Health Centre to elicit feedback prior to online distribution of the survey to clinicians in other Canadian hospitals.

The paper-based survey provided respondents the opportunity to make comments about the survey itself and suggestions for improvement. No changes were recommended by the local respondents to improve the survey content itself. Thus, the survey was distributed nationally.

\section{Development of the web-based survey}

The web-based survey was developed using OPINIO, a licensed and secure online survey software that is freely available to researchers at Dalhousie University.

The web-based survey was distributed via email to all practicing pediatric hematologist-oncologists in Canada and their subspecialty trainees. The email addresses were collected from publicly available departmental websites by the PI. The only contact information available on departmental websites for pediatric hematologyoncology subspecialty residents were for chief subspecialty residents or program administrators, so these email addresses were used. Pediatric hematologist-oncologists in Canada and their subspecialty trainees who were personally known to the author(s) were also contacted by email.

Respondents were initially given 2 weeks to respond to the survey online, with a reminder email sent after 1 week. A second email was sent approximately one month later to promote more survey responses. After the last survey deadline, responses were exported from OPINIO software to an Excel spreadsheet for analysis. Survey results are anonymous and contain no identifying information.

Ethics

This study was approved by the local Research Ethics Board (File Number: 1025717).

\section{Results}

Survey response rates 
In Canada, there are 16 hospitals that are affiliated with Children's Oncology Group (COG). We received survey responses from at least one clinician at 15 of these 16 hospitals.

We contacted a total number of 101 pediatric hematology-oncology faculty members who treat patients with leukemia and lymphoma. We received a total of 51 responses, yielding a survey response rate of $41.5 \%$ for faculty. We also received a total of 9 responses from subspecialty residents in pediatric hematology-oncology from across Canada.

\section{Data Analysis}

Descriptive statistics were analyzed by OPINIO software and available for analysis, including absolute and relative frequencies for each response.

\section{Missing responses}

There were 4 missing responses to question 6, 1 missing response to questions 12, 14 and 19 for a total of 7 missed responses.

Demographics of respondents

Survey respondents represented 15 Canadian hospitals, with at least one clinician representative of each Canadian region, and a range of clinical experience. Detailed demographic information for survey respondents is described in Table 1. We received the most responses from Western Canada (44\%) and Eastern Canada $(33.3 \%)$.

Subspecialty residents in pediatric hematology-oncology represented $17.6 \%$ of survey respondents ( 2 in year one, 4 in year 2, and 3 in year 3 or higher) while the remaining $82.4 \%$ were faculty with a range of experience: 12 respondents were faculty for $<5$ years, 15 for 5-15 years, 12 for 15-30 years and 3 for 30 or more years. Only 3 respondents (6\%) reported being a clinician scientist spending $>50 \%$ of their time on research.

\section{Measurement of pegaspargase levels}

All but 3 respondents reported using primarily pegasparaginase intravenously at their centre. Out of the 3 that were unsure, 2 respondents were from Eastern Canadian centres, and one from a Western Canadian centre.

Twenty respondents (39.2\%) reported routinely measuring levels. These respondents were mostly from Eastern and Atlantic Canada. The most common reasons for not routinely measuring asparaginase levels was that respondents were unsure how to use levels clinically (relative frequency $=25.5 \%$ ), were unsure if this was required (relative frequency $=21.6 \%$ ) or felt there was no centre/laboratory available to send the samples for analysis (relative frequency $=19.6 \%$ ). Other reasons included cost (relative frequency $=11.8 \%$ ) and overall lack of knowledge regarding how and when to send levels (relative frequency $=13.7 \%$ for each).

At hospitals that measure asparaginase levels, levels are reviewed primarily by clinicians (including subspecialty residents, nurse practitioners and physicians) (relative frequency $=45.1 \%$ ), but also by pharmacists (relative frequency $=19.6 \%$ ).

When asked regarding timing of levels, Day 6-7 was the most popular selection with a relative frequency of $29.4 \%$. Day 0 was selected with a relative frequency of $9.8 \%$, Day 2 with a relative frequency of $1.9 \%$ and Day 14 with a relative frequency of $9.8 \%$ (see Table 2 ).

Measurement of Erwinia asparaginase levels

Most respondents do not routinely measure asparaginase levels in patients receiving Erwinia asparaginase (52.9\%). 9 respondents measure asparaginase levels in these patients 17.6\%)

\section{Modification of asparaginase therapy}

When asked about what scenarios would prompt them to modify therapy from pegaspargase to Erwinia asparaginase, pegaspargase levels below threshold on any occasion was chosen as a response $11.1 \%$ of the 
time. Grade 4 infusion reactions was chosen $30.6 \%$ of the time, while Grade 3 infusion reactions were chosen $29.6 \%$ of the time, Grade 2 infusion reactions $12.9 \%$ of the time and Grade 1 infusion reactions only $0.9 \%$ of the time [4]. Other reasons for modification of therapy from pegaspargase to Erwinia asparaginase were provided as pancreatitis and thrombosis.

Most respondents (47.1\%) indicated modifying therapy from pegaspargase to Erwinia asparaginase in 10$25 \%$ of patients treated. $31.4 \%$ of respondents indicated modifying therapy in $<10 \%$ of patients treated. Only $5.9 \%$ of respondents indicated modifying therapy in $20-50 \%$ of patients treated, while one respondent has never modified therapy and $7(13.67 \%)$ were unsure.

\section{Prevalence of silent inactivation}

$13.7 \%$ respondents indicated that they have never treated a patient with silent inactivation, $19.6 \%$ had treated $<10 \%$ of patients with silent inactivation and $66.7 \%$ were unsure how many patients they have treated with silent inactivation.

\section{Management of hypersensitivity reactions}

Seventeen respondents (33.3\%) indicated that they routinely administer pre-medications before all doses of pegaspargase, even if the patient has not experienced an infusion-related reaction. These respondents were from the Hospital for Sick Children, McMaster University, British Columbia Children's Hospital and the IWK Health Centre.

8 respondents $(15.7 \%)$ indicated they have never administered pre-medication before pegaspargase. Five respondents $(9.8 \%)$ indicated they would only administer pre-medications before pegaspargase if checking asparaginase levels. When asked about which grades of infusion reactions would prompt the routine administration of pre-medication, a history of a Grade 1 infusion-related reaction was chosen as a response $13.9 \%$ of the time, Grade 2 reaction $23.1 \%$ of the time, and Grade 3 and 4 reactions both at $6.2 \%$ of the time (presumably because these patients would have been switched to Erwinia asparaginase).

The most common premedication used was identified as antihistamine (H1 receptor antagonists) with a relative frequency of $60.8 \%$, followed by steroids (relative frequency $=25.5 \%$ ), $\mathrm{H} 2$ receptor antagonists (relative frequency $=23.5 \%$ ), anti-emetics (relative frequency $=19.6 \%$ ) and antipyretics (relative frequency $=19.6 \%$ ).

$47 \%$ of respondents indicated they would "re-challenge" pegaspargase in a patient with a previous history of an infusion-related reaction, but only if the patient had a history of a Grade 1 or 2 infusion-related reaction. $25.5 \%$ of respondents indicated they would only consider a "re-challenge" if the initial reaction was Grade 1. $17.6 \%$ of respondents indicated they would rechallenge even if the patient had a history of a Grade 3 infusion-related reaction, while $7.8 \%$ of respondents indicated they would never attempt to administer IV pegasparagase again to that patient, regardless of the Grade of infusion reaction.

\section{Discussion}

This is the first survey to measure the practice of asparaginase activity monitoring and the management of reactions to pegaspargase across pediatric oncologists and trainees in Canada. Respondents represented 15 of 16 COG-affiliated centres with a variety of clinical experience.

Clinicians primarily use IV pegaspargase which is consistent with COG protocols. The only Canadian centres that routinely measure asparaginase levels are the Hospital for Sick Children, McMaster University, CHU Sainte-Justine, CHU de Quebec-Universite Laval and the IWK Health Centre. Most respondents from these centres indicated they draw levels at Day 0 and/or Day 6-7, which is consistent with expert recommendations $[5,6]$.

There is a range of target asparaginase levels across respondents, but all target a level of at least $0.1 \mathrm{IU} / \mathrm{mL}$, which is concordant with standards of practice (SOP) at the Hospital for Sick Children and the IWK Health Centre $[5,6]$ and with consensus guidelines, all of which suggest that a trough level $<0.1 \mathrm{IU} / \mathrm{mL}$ is 
subtherapeutic $[5,6]$. Most respondents noted that $<10 \%$ of patients in their practice have experienced low levels, which is consistent with the cited prevalence of this phenomenon $(8 \%)$ [2].

The most common reasons to modify therapy from pegaspargase toErwinia asparaginase was for a Grade 3-4 infusion reaction or for asparaginase levels that are subtherapeutic, which is consistent with most consensus guidelines [6]. COG protocols state that discontinuation of pegaspargase is recommended for Grade 2 or higher reactions, but only a Grade 3 or higher reaction mustswitch asparaginase preparations [3]. The National Comprehensive Cancer Network (NCCN) clinical practice guidelines also support switching asparaginase preparations in event of a Grade 3 reaction or higher [5,7-9]. More recent COG protocols include a severity scale suggestive that severe reactions warrant a switch to Erwiniaasparaginase but leave the definition of severe to the treating institution $[10,11]$.

The rate of hypersensitivity reactions to pegaspargase is reported to be as high as $30 \%$ [7]. Despite this high prevalence, over $70 \%$ of respondents stated they do not routinely administer pre-medications before all doses of pegaspargase. Evidence suggests that premedication can significantly reduce the rate of hypersensitivity reactions and although premedication for all patients receiving pegaspargase is done routinely in adult ALL protocols, this is not mandated in pediatric protocols [12]. There is historical concern that premedication can mask the development of a clinically relevant reaction that prior to the availability of routine asparaginase assays was used as a sign of anti-asparaginase antibody development and a required switch toErwinia asparaginase. All patients at the IWK receive premedication prior to all asparaginase doses whether or not they have previously experienced a reaction. These pre-medications include acetaminophen, ondansetron, cetirizine, diphenhydramine, famotidine $+/$ - steroid.

One of the limitations of this study was the inability to contact every practicing pediatric oncologist and subspecialty trainee in Canada. However, the PIs personally reached out to every institution so that maximum representation was sought and bias was minimized. Several institutions indicated that their point person for leukemia has/have filled out the survey. Thus, we believe that optimum attempts were made to minimize the bias and optimize response rate. Other limitations inherent to survey studies include the risk of recall bias and misinterpretation of the questions.

\section{Conclusion}

Up to $30 \%$ of patients receiving pegaspargase will experience a clinically apparent reaction, while only $8 \%$ of patients will experience SI. The only reliable way to distinguish between these phenomena is by measuring an asparaginase level. In patients with a CAR and therapeutic asparaginase level, the switch to Erwinia asparaginase can usually be avoided. The only evidence-based indication to switch asparaginase formulations from pegaspargase to Erwiniaasparaginase is in the context of a severe hypersensitivity reaction or in the event of an asparaginase trough level of $<0.1 \mathrm{IU} / \mathrm{mL}[3]$.

The Hospital for Sick Children has been routinely measuring asparaginase levels in all patient since 2016, and they recently published a study that concluded that the appropriate use of asparaginase level monitoring avoided a switch to Erwinia asparaginase in 3 of 16 patients [3]. In our study, clinicians reported switching patients from pegaspargase to Erwinia asparaginase up to $25 \%$ of the time, which is possibly more than required given that the prevalence of SI is likely $<10 \%$. At the IWK, nearly $100 \%$ of asparaginase levels were supratherapeutic regardless of the presence of a CAR, suggesting that the rate of SI is very low and that we likely switch more patients to Erwinia asparaginase than we should [13].

Despite this evidence, this study confirmed that the majority of Canadian centres do not routinely measure asparaginase levels due to perceived barriers to using and interpreting asparaginase levels. There are now CLIA-certified laboratories that offer asparaginase level testing on serum/plasma specimens in Canada.

Even within Canadian hospitals that do routinely measure asparaginase levels, there was some variation regarding the timing of levels and what levels to target. This indicates either a lack of a clear institutional SOP and/or a lack of education and/or a lack of a national consensus.

With the impending global shortage of Erwiniaasparaginase, it is imperative that the pediatric oncology 
community use asparaginase levels to determine when a switch from pegaspargase to Erwinia asparaginase is justified, as this will likely lead to fewer patients requiring a switch to Erwiniaasparaginase and therefore conserve its supply. It will also allow more children to be re-challenged and receive adequate asparaginase treatment. The authors of this paper feel it is important to develop a pan-Canadian practice guideline to asparaginase activity monitoring that can be implemented at each centre to help circumvent some of the perceived barriers to asparaginase activity monitoring and improve asparaginase treatment in children.

\section{Conflict of Interest Statement}

The authors do not have any conflict(s) of interest to declare.

\section{Acknowledgements}

The authors wish to acknowledge all those who participated in this study by completing the survey.

\section{References}

1. Nadeem K et al. Clinical decisions following implementation of asparaginase activity monitoring in pediatric patients with acute lymphoblastic leukemia: Experience from a single-center study. Pediatric Blood and Cancer 2019;e28044:1-7.

2. Tong WH, Pieters R, Kaspers GJL, et al. A prospective study on drug monitoring of PEGasparaginase and Erwinia asparaginase and asparaginase antibodies in pediatric acute lymphoblastic leukemia. Blood. 2014;123:2026-2034. Wacker P et al. Allergic Reactions to E.Coli L-Asparaginase Do Not Affect Outcome in Childhood B-precursor Acute Lymphoblastic Leukemia. A Children's Oncology Group Study. J Pediatr Hematol Oncol 2007;29(9):627-632.

3. Marini et al. A single-center multidisciplinary approach to managing the global Erwinia asparaginase shortage. Leukemia \& Lymphoma 2019;60(12):2854-2868.

4. U.S Department of Health and Human Services. National Cancer Institute Division of Cancer Treatment and Diagnosis. Common Terminology Criteria for Adverse Events (CTCAE) Version 5.0 Published: November 27, 2017. Available at: https://ctep.cancer.gov/protocoldevelopment/electronic_applications/ctc.htm\#ctc_50

5. Salzer $\mathrm{W}$ et al. (2017). Asparaginase activity levels and monitoring in patients with acute lymphoblastic leukemia. Leukemia and Lymphoma, 1-10.

6. Van der Sluis IM et al. Consensus expert recommendations for identification and management of asparaginase hypersensitivity and silent inactivation. Haematologica 2016;101(3):279-285.

7. Marini BL, Perissinotti AJ, Bixby DL, et al. Catalyzing improvements in ALL therapy with asparaginase. Blood Rev. 2017;31:328-338.

8. Burke MJ, Rheingold SR. Differentiating hypersensitivity versus infusion-related reactions in pediatric patients receiving intravenous asparaginase therapy for acute lymphoblastic leukemia. Leuk Lymphoma. 2017;58:540-551.

9. Network NCC. Clinical Practice Guidelines in Oncology - Acute Lymphoblastic Leukemia. Version 1.2018. 2018.

10. Gupta S, Rau R et al. A Phase 3 Trial Investigating Blinatumomab (IND\# 117467, NSC\# 765986) in Combination with Chemotherapy in Patients with Newly Diagnosed Standard Risk or Down syndrome B-Lymphoblastic Leukemia (B-ALL) and the Treatment of Patients with Localized B-Lymphoblastic Lymphoma (B-LLy). Children's Oncology Group.

11. O'Brien M, McNeer J et al. A Phase 3 Randomized Trial of Inotuzumab Ozogamicin (IND\#:133494, NSC\#: 772518) for Newly Diagnosed High-Risk B-ALL; Risk-Adapted Post-Induction Therapy for High-Risk B-ALL, Mixed Phenotype Acute Leukemia, and Disseminated B-LLy

12. Young D, Bowen C, Arwood N, et al. Universal Pretreatment for Asparaginase-Based Therapy Should be Standard of Care. ASPHO 2018. 2018 (Abstract 2024). [cited 2018 Oct 17]. Available from: https://aspho.planion.com/Web.User/AbstractDet?ACCOUNT1/4 ASPHO\&ABSID1/413563\&CONF1/4AM18\&ssoOverride1/4O- FF\&CKEY.

13. Pike M, Kulkarni K, MacDonald T. Supratherapeutic levels of asparaginase: therapeutic drug moni- 
toring experience from the Maritimes, Canada. Leukemia and Lymphoma 2019;60(9):2312-2315.

\section{Hosted file}

Table 1.pdf available at https://authorea.com/users/397927/articles/510667-asparaginaseactivity-monitoring-and-management-of-asparaginase-hypersensitivity-reactions-in-canada

Hosted file

Table 2.pdf available at https://authorea.com/users/397927/articles/510667-asparaginaseactivity-monitoring-and-management-of-asparaginase-hypersensitivity-reactions-in-canada 